\title{
Comparative Analysis of Mobile Ad Hoc Network Routing Protocols Simulation
}

\author{
Xiaoyan Wei \\ School of Software \\ Xiamen University \\ Xiamen, Fujian, 361005, China \\ yingxie.wxy@gmail.com
}

\begin{abstract}
A Mobile Ad Hoc Network(MANET) is a popular research topic. In this paper, we first describe characteristics of MANET, proactive and reactive routing protocols. Then AODV and DSDV, which are widely investigated in Ad Hoc network are introduced. We use simulation tool NS-2 to design three experiments to evaluate AODV and DSDV based on measure such as the packet drop rate, delays and average throughout. According to experimental results, conclusions and improvement suggestions are given to help further develop and optimize routing protocols.
\end{abstract}

Keywords-MANET; AODV; DSDV; NS-2

\section{INTRODUCTION}

With the continuous development of network technology, the wireless network gets more and more attention as its convenience and scalability. A mobile ad hoc network (MANET) is a self-configuring infrastructureless network of mobile devices connected by wireless[1]. The growth of laptops and Wi-Fi wireless networking have made MANETs a popular research topic.

Ad hoc research focus on routing protocols[2], quality of service[3], MAC protocols[4], energy consumption[5], node mobility management[6], security[7], etc. Routing protocols are the most concentrated part of the Ad Hoc research. Many academic papers evaluate protocols and their abilities, assuming varying degrees of mobility within a bounded space, usually with all nodes within a few hops of each other. Routing protocols are classified as proactive (example DSDV, OLSR) and reactive (example DSR, AODV) on the basis of their routing methods[8]. Proactive protocols determine routes independent of traffic pattern. Traditional link-state and distance-vector routing protocols are proactive. Reactive protocols maintain routers only if needed.

\section{RELATED WORK}

Proactive and reactive protocols have their own advantages and disadvantages. This paper chooses DSDV and AODV to compare and evaluate their performance use different nodes.

\section{A. DSDV:Destination-Sequenced Distance-Vector Routing}

DSDV is an improvement on the classical Bellman-Ford routing mechanism[9]. It update route table in two ways. One is full dump, all available routing info and multiple network protocol data units. The other is incremental packets, router relay info between two full dumps. When using
DSDV, each node maintains a routing table which stores. Sequence numbers used to avoid formation of loops. A destination sequence number that is created by the destination itself.

Each node periodically forwards the routing table to its neighbors. Each node increases and appends its sequence number when sending its local routing table. This sequence number will be attached to route entries created for this node. Hosts perform periodic and triggered updates, issuing a new sequence number. Sequence number indicates the "freshness" of a route. Routes with more recent sequence numbers are preferred for packet forwarding. If same sequence number, one having smallest metric used.

\section{B. AODV: Ad Hoc On-Demand Distance Vector Routing}

AODV typically minimizes the number of required broadcasts by creating routes on a demand basis[10], as opposed to maintaining a complete list of routes. When route moves, its upstream neighbor notices the move and propagates Link Failure Notification Message (LFNM) to each of its active upstream neighbors to inform erasure. Then Source node reinitiate route discovery. Hello Message is sent to maintain the local connectivity in one node's neighborhood. AODV attempts to improve on DSR by maintaining routing tables at the nodes, so that data packets do not have to contain routes.

AODV assumes symmetric (bi-directional) links. When a node re-broadcasts a Route Request, it sets up a reverse path pointing towards the source. When the intended destination receives a Route Request, it replies by sending a Route Reply. Route Reply travels along the reverse path setup when Route Request is forwarded. To determine whether the path known to an intermediate node is more recent, destination sequence numbers are used. The likelihood that an intermediate node will send a Route Reply when using AODV.

\section{SiMULATION EXPERIMENT PROCESS}

There are several ways to study protocols. One solution is the use of simulation tools like NS-2[11]. NS-2 is a discrete event simulator targeted at networking research. It provides substantial support for simulation of TCP, routing, and multicast protocols over wired and wireless (local and satellite) networks.

The emulation environment settings are as follows. Both moving width and length are $1200 \mathrm{~m}$. Maximum moving speed is $20 \mathrm{~m} / \mathrm{s}$, and average moving speed is $10 \mathrm{~m} / \mathrm{s}$. We use 
constant bit stream CBR to send one packet, whose length is 512 byte, one second. Time is set to 100 seconds. The number of nodes are 8, 16, 32, 64(see Fig. 1).

NS-2 simulation process as shown in Fig. 2.

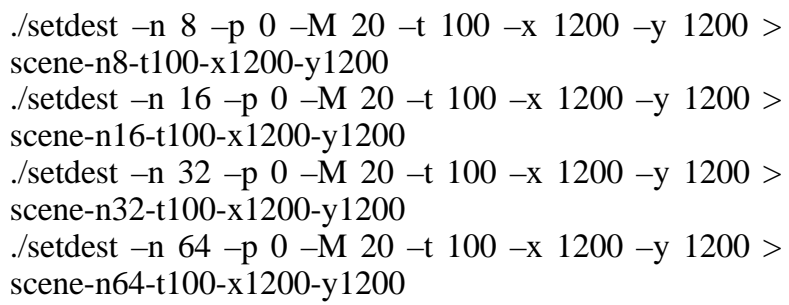

Figure 1. Environment buliding

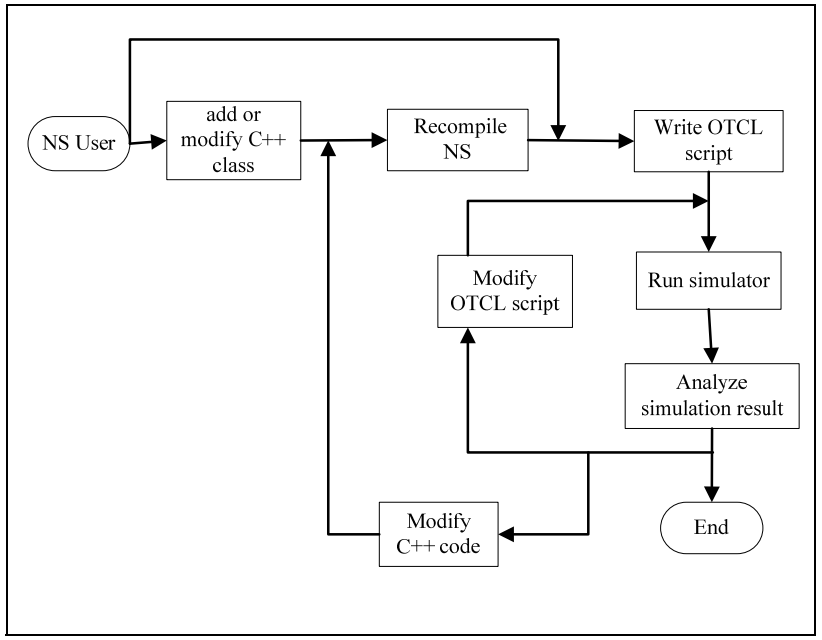

Figure 2. NS-2 simulating process

After building platform and environment, we need to generate the throughout load, write TCL script and AWK script. We use metric such as packet drop rate, time delay and network throughout with different number of nodes to evaluage performance of AODV and DSDV. Experimental results are shown in Tables I-III.

TABLE I. PACKet Drop Rate Of Aodv(Left) And Dsdv(Right)

\begin{tabular}{|c|c|c|c|}
\hline $\begin{array}{c}\text { Number of } \\
\text { Nodes }\end{array}$ & $\begin{array}{c}\text { Number of } \\
\text { Packets Sent }\end{array}$ & $\begin{array}{c}\text { Number of } \\
\text { Packets Received }\end{array}$ & $\begin{array}{c}\text { Packet Drop } \\
\text { Rate }\end{array}$ \\
\hline 8 & $358 / 347$ & $50 / 30$ & $0.8603 / 0.9135$ \\
\hline 16 & $552 / 564$ & $185 / 45$ & $0.6649 / 0.9202$ \\
\hline 32 & $563 / 556$ & $483 / 165$ & $0.1421 / 0.7032$ \\
\hline 64 & $556 / 573$ & $415 / 73$ & $0.2536 / 0.8726$ \\
\hline
\end{tabular}

TABLE II. Time Delay of Aodv(LeFt) AND DSDV(Right)

\begin{tabular}{|c|c|c|c|}
\hline $\begin{array}{c}\text { Number } \\
\text { of Nodes }\end{array}$ & $\begin{array}{c}\text { Maximum } \\
\text { Delay(s) }\end{array}$ & $\begin{array}{c}\text { Minimum } \\
\text { Delay(s) }\end{array}$ & $\begin{array}{c}\text { Average } \\
\text { Delay(s) }\end{array}$ \\
\hline 8 & $9.71 / 0.25$ & $0.01 / 0.01$ & $1.27 / 0.01$ \\
\hline 16 & $16.67 / 4.12$ & $0.01 / 0.01$ & $3.02 / 0.17$ \\
\hline 32 & $11.78 / 4.98$ & $0.01 / 0.01$ & $0.49 / 0.10$ \\
\hline
\end{tabular}

\begin{tabular}{|l|l|l|l|}
\hline 64 & $20.72 / 2.35$ & $0.01 / 0.01$ & $0.84 / 0.03$ \\
\hline
\end{tabular}

TABLE III. Average Throughout Aodv(LeFt) AND Dsdv(Right)

\begin{tabular}{|c|c|c|}
\hline Number of Nodes & $\begin{array}{c}\text { Routing Protocol } \\
\text { Throughout(KB/s) }\end{array}$ & $\begin{array}{c}\text { CBR } \\
\text { Throughout(KB/s) }\end{array}$ \\
\hline 8 & $0.08 / 0.06$ & $0.26 / 0.16$ \\
\hline 16 & $0.34 / 0.19$ & $0.96 / 0.23$ \\
\hline 32 & $0.76 / 0.84$ & $2.51 / 0.86$ \\
\hline 64 & $3.05 / 3.69$ & $2.16 / 0.38$ \\
\hline
\end{tabular}

In TABLE I, we can that with the increase of nodes(From eight to Thirty-two),there has a pretty significant decline in packet drop rate. When the number of nodes increases from thirty-two to sixty-four, packet drop rate rises a little. Networks running DSDV protocol are much smaller in time of delay than those running AODV protocol, which as shown in TABLE II.

Both AODV and DSDV routing protocol rise significantly in network throughout, while behave widely different in CBR Throughout with the increase of nodes. Networks running AODV protocol have large rise in CBR throughout, while those running DSDV protocol don't have much change(see TABLE III).

\section{COMPARISON AND EVALUATION}

By using tool gnuplot, we draw contrast diagram to compare and evaluate AODV and DSDV, which as shown from Fig. 3 to Fig. 5.

We can get a conclusion as follows. In packet drop date, AODV protocol is obviously superior to the DSDV protocol. The average delay time of DSDV protocol is better than that of AODV protocol, and the change of delay is less; AODV protocol spends less overhead than DSDV protocol in average throughout. It has a huge advantage in data throughout at the same time.

Overall, AODV protocol has a low packet loss ratio and large data throughout. On the contrary, DSDV protocol has a short average delay. In addition, when node number is small, the environment density is small too. It's extremely difficult to form a network between these nodes.

As a result, formed network has a bad performance in delay and packet loss. When nodes number increases, the network overhead increases obviously and data throughout decrease at the same time. So AODV is suitable for occasions whose scales are not big and packet transmission rate are low. When the network's nodes move slowly, it suits to run DSDV protocol. 


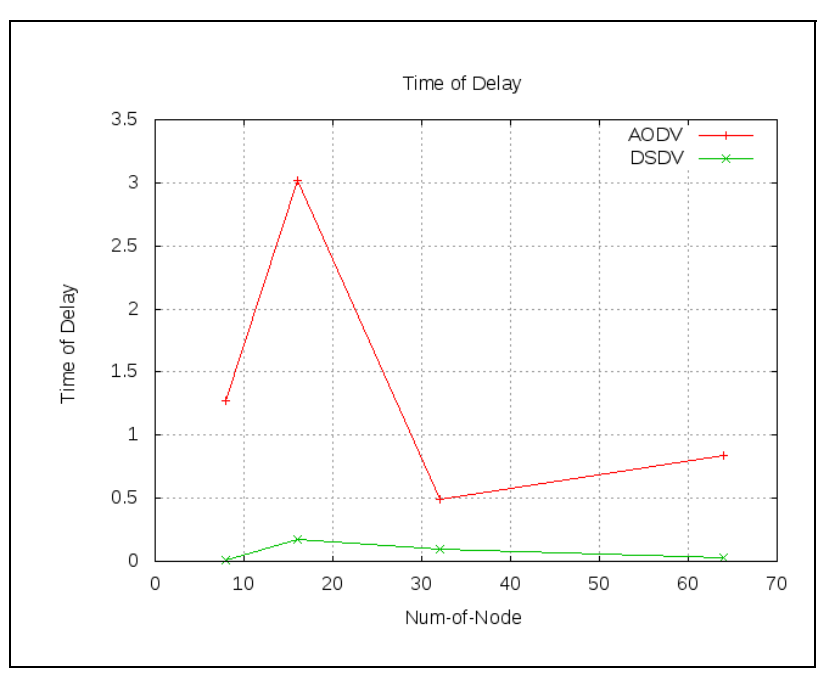

Figure 3. Comparation of delay

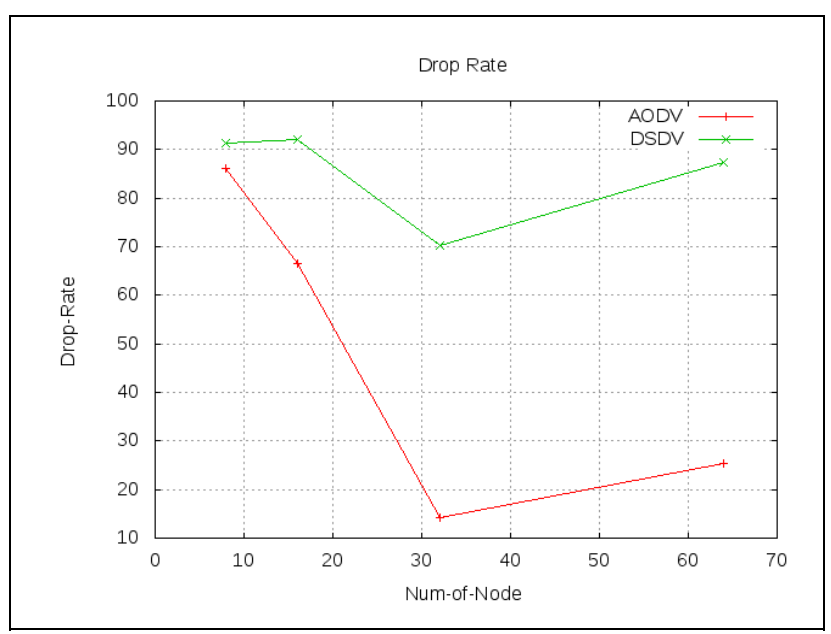

Figure 4. Comparation of drop rate

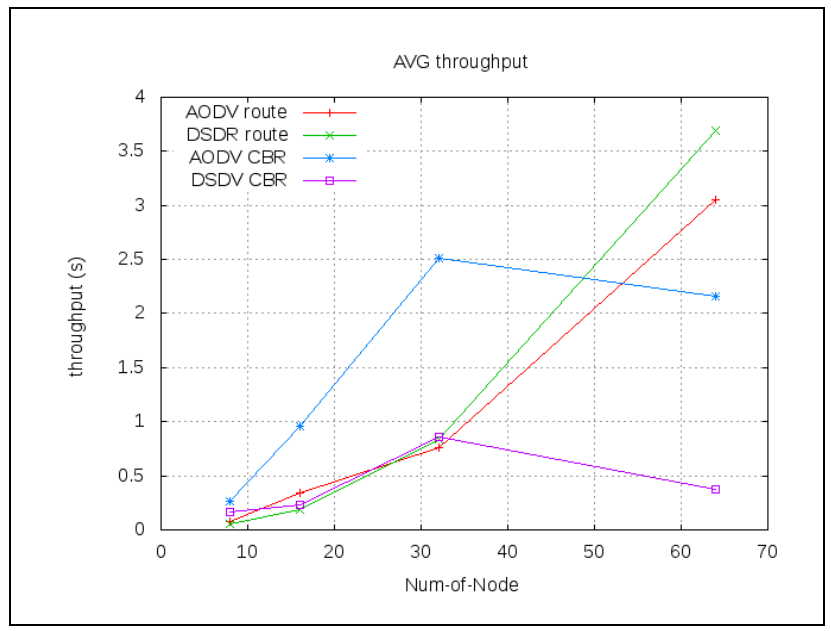

Figure 5. Comparation of average throughout

\section{IMPROVEMTN SUGGESTIONS}

We can see from the result of the comparison that different routing protocols have their own applications, advantages and disadvantages.

DSDV algorithm is a table-driven routing protocol. It makes an improvement to classical Bell-Ford routing algorithm. It has poor scalability, a low packet transmission rate and high overhead. It transmissions only in a single path. For these disadvantages, multi-path transmission mode[12] can be used to improve DSDV algorithm on the basis of fourth-generation communication. This mode transmits two different packets in two different paths simultaneously and can use the parallel network resources more effectively. Through this we can get a good throughout performance gain.

AODV belongs to the on-demand routing algorithm. It is a combination of on-demand type and table-driven type, with the advantages of these two ideas. AODV has a low overhead of proceeding and storage and can make a rapid response to link state change.

By using the serial number, AODV can be guaranteed not to form a routing ring to avoid infinite counting problem[13]. But AODV protocol is based on the assumption that networks only existing bidirectional links. If networks exist unilateral links, the performance of AODV will be influenced badly, even fatally. In response to these shortcomings, AODV-BL[14] and HELLO mechanism[15] are put forward to improve AODV.

Common disadvantage of AODV and DSDV is when the number of nodes increases, occupancy to links increases too, which exacerbates the burden of networks and limits the effective data flow. We can improve algorithms respectively, but a better solution is to combine with prior and reactive routing protocols to form a hybrid routing. It can reduce the cost of the routing protocol under the premise of minimizing packet delay.

References 16 presents a hierarchical routing algorithm based on the clustering structure[16]. Priori routing algorithm is used in clusters. It searches routing on demand and adjusts the size of the cluster according to dynamic change of networks adaptively. This algorithm absorbs advantages of two routing algorithms, which has high efficiency and strong adaptability.

Furthermore, Genetic Algorithm(GA) and Ant Colony Optimization(ACO) can also be used in the Ad Hoc routing protocols improvement. In references 17 , it presents a wireless routing algorithm based on GA[17]. This algorithm is simple, short-time and easy to obtain alternative paths. References 18 applies ACO[18] to solve the problem that paths disconnect as propagation distance limit, which improve the efficiency of AODV algorithm.

\section{CONCLUSION}

The trend of communication development is broadband, mobile and intelligent. Personal communication is the highest goal of human communication. In this paper, we use simulation tool NS-2 to design three experiments to evaluate AODV and DSDV. Then we give improvement suggestions to help further develop and optimize routing protocols 
according to experimental results. Routing protocols of ad hoc has been a hot area of research for several years. But it is complicated and still need long-term effort and exploration to achieve satisfactory routing algorithm and mechanism.

\section{ACKNOWLEDGMENT}

The author thanks Prof. Liao Minghong and Mr. Lin Xinhong for their help to conduct a survey amongst mobile ad hoc network and simulation experiments.

\section{REFERENCES}

[1]R. J. Ramanathan R, "A Brief Overview of Mobile Ad hoc Networks:Challenges and Direction," IEEE Communications Magazine, vol. 40, pp. 20-23, 2002.

[2]B. P. Stefaan Seys, "ARM: Anonymous Routing Protocol for Mobile Ad hoc Networks," International Journal of Wireless and Mobile Computing pp. 145-155, Monday, October 12, 20092009.

[3]A. A. a. W. Zhuang, "Statistical QoS Routing for EEE 802.11 Multihop Ad Hoc Networks," IEEE TRANSACTIONS ON WIRELESS COMMUNICATIONS, vol. 8, pp. 1542-1552, 3,2009 2009.

[4]N. S. a. T. Fujii, "A MAC Protocol for Multi-Packet Ad-hoc Wireless Network Utilizing Multi-Antenna " presented at the Consumer Communications and Networking Conference,2009. CCNC 2009. 6th IEEE, Las Vegas, NV 2009.

[5]X. W. Xiaojing Xiang, Zhehua Zhou, "An Efficient Geographic Multicast Protocol for Mobile Ad Hoc Networks," presented at the Proceedings of the 2006 International Symposium on a World of Wireless, Mobile and Multimedia Networks, 2006.

[6]A. A. Jiazi Yi, Sylvain David,Benoit Parrein,, "Multipath optimized link state routing for mobile ad hoc networks," Ad Hoc Networks, vol. 9, pp. 2847, January 20112011.

[7]M. C. Mohammad O. Pervaiz, and JieWu, "Routing Security in Ad Hoc Wireless Networks," Department of Computer Science and Engineering, pp. 117-142, 2010.
[8]Z. A. ZHAO Ggenx, SUN Wei, "An Overview of Ad Hoc Routing Protocols," Inner Mongolia Scientech and Economy, vol. 10, pp. 45-46, 2003.

[9]P. C. a. B. P, "Highly dynamic destination-sequenced distance-vector routing(DSDV) for mobie Computer," Computer Communication Review, vol. 24, pp. 234-244, 1994.

[10]R. E. Perkins C, and Das S R. (November,2000) Ad hoc On-demand Distance Vector(AODV) Routing. IETF Intent draft,draft-ietf-manet-adov07.txt.

[11]T. U. o. S. California. NS official website.[EB/OL]. http://www.isi.edu /nsnam/ns/index.html.

[12]B. X. ZHENG Chuangming, "An Improvement of DSDV Based on Fourth Generation of Communication Network," Commercial Modernization, pp. 26-28, 2009.

[13]M. MIisrar, "Performance Comparison of AODV /DSR On-demand Routing Protocols for Ad hoc Networks in Consrained Situation " presented at the IEEE International Conference, 2005.

[14]D. H. Megat Zuhairi, "A Simulation Study on the Impact of Mobility Models on Routing Protocol Performance With Unidirectional Link Presence " in Information Networking (ICOIN), 2011 International Conference, Barcelona, 2011, pp. 335-340.

[15]T. Kim, B. Kang, E. K. Park, and S. Tak, "Integrating real-time hybrid task scheduling into a sensor node platform," presented at the Proceedings of the 2010 ACM Symposium on Applied Computing, Sierre, Switzerland, 2010.

[16]Y. Wei-dong, "Ad Hoc Network Clustering Algorithm Considering Node Energy Status," Computer Engineering, vol. 36, pp. 119-122, 2010.

[17]W. J. WANG Zhen-chao, JING Xin, "Research on Multipath Routing Based on Genetic Algorithm," Computer Engineering, vol. 37, pp. 197-199, 10,2011 2011.

[18]R. G. Jyoti Jain, T.K. Bandhopadhyay, "Ant Colony Algorithm in MANET-Local link repairing of AODV," presented at the Electronics Computer Technology (ICECT), 2011 3rd International Conference Kanyakumari 2011. 